\title{
Network-Oriented Radiation Monitoring System (NORMS)
}

\author{
IEEE Nuclear Science Symposium
}

\author{
Rahmat Aryaeinejad \\ David F. Spencer
}

The INL is a

U.S. Department of Energy

National Laboratory

operated by

Battelle Energy Alliance

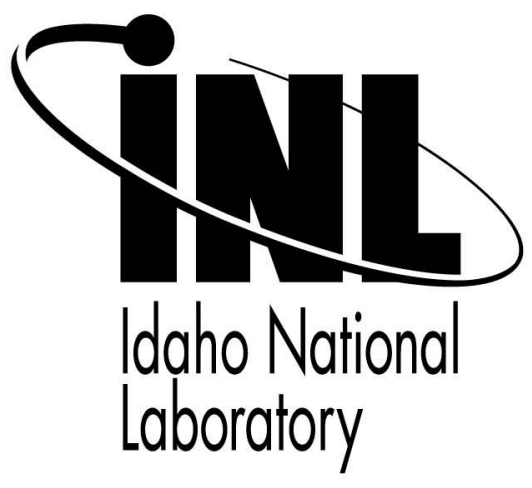

This is a preprint of a paper intended for publication in a journal or proceedings. Since changes may be made before publication, this preprint should not be cited or reproduced without permission of the author. This document was prepared as an account of work sponsored by an agency of the United States Government. Neither the United States Government nor any agency thereof, or any of their employees, makes any warranty, expressed or implied, or assumes any legal liability or responsibility for any third party's use, or the results of such use, of any information, apparatus, product or process disclosed in this report, or represents that its use by such third party would not infringe privately owned rights. The views expressed in this paper are not necessarily those of the United States Government or the sponsoring agency. 


\title{
Network-Oriented Radiation Monitoring System (NORMS)
}

\author{
Rahmat Aryaeinejad and David F. Spencer
}

\begin{abstract}
We have developed a multi-functional pocket radiation monitoring system capable of detecting and storing gamma ray and neutron data and then sending the data through a wireless connection to a remote central facility upon request. The device has programmable alarm trigger levels that can be modified for specific applications. The device could be used as a stand-alone device or in conjunction with an array to cover a small or large area. The data is stored with a date/time stamp. The device may be remotely configured. Data can be transferred and viewed on a PDA via direct connection or wirelessly. Functional/bench tests have been completed successfully. The device detects low-level neutron and gamma sources within a shielded container in a radiation field of $10 \mathrm{uR} / \mathrm{hr}$ above the ambient background level.
\end{abstract}

\section{INTRODUCTION}

$\mathrm{T}$ he U.S. nuclear storage facilities are definitely in need of new radiation detection monitoring technologies, which can improve safeguards to protect against a variety of unwanted events, such as theft of nuclear weapons and special nuclear materials and radiological sabotage. Our goal was to develop and commercialize cheaper, faster, and integrated smart radiation monitoring systems that can be implemented at nuclear facilities of the U.S. Department of Energy (DOE) and the National Nuclear Security Administration (NNSA), as well as throughout the international safeguards community (IAEA).

The Network-Oriented Radiation Monitoring System (NORMS), which has been developed at Idaho National Laboratory (INL), is a detection device capable of operating in a networked or a stand-alone configuration. It is capable of detecting both neutrons and gamma rays simultaneously, comparing the detected levels against configurable alarm setpoints, and then notifying a remote or a local location, respectively. Although the detection system can be used as a stand-alone unit, it is primarily designed to be integrated with wireless network radiation monitoring systems.

Traditionally, two separate detectors are used, one for gamma rays and the other for neutrons. This makes the detection system heavy, bulky and difficult to carry. The

Manuscript received October 28, 2003. This work was supported in part by the U.S. Department of Energy under DOE Idaho Operations Office Contract DE-AC07-05ID14517.

R. Aryaeinejad is with the Idaho National Laboratory, Idaho Falls, ID 83415-2114 USA (e-mail: Rahmat.Aryaeinejad@inl.gov, telephone: 208-5261670).

D. F. Spencer is with the Idaho National Laboratory, Idaho Falls, ID 83415-3779 USA (e-mail: David.Spencer@inl.gov, telephone: 208-5261489). sensors we utilized are based on a combination of ${ }^{6} \mathrm{Li} /{ }^{7} \mathrm{Li}-$ loaded glass scintillators to detect neutrons and gamma rays simultaneously and instantly. The detection system is a modified version of a previously developed neutron/gamma device at the INL [1]-[2]. Because the ${ }^{6} \mathrm{Li}$ detector is in solid form, a thickness of only few millimeters is enough to stop thermal neutrons [3]. Therefore, much smaller, lighter, less expensive and more sensitive detection systems can be fabricated. The device is intended to be used to detect the presence of neutron and gamma-ray radiation fields and their relative intensities. It can also be used to find the location of the radioactive source. The sensor operates on one rechargeable Li-ion battery. The high voltage to the detectors is provided using a small Cockroft-Walton circuit board. At this time there are few other commercially available single devices [4]-[5] that can detect both gamma and neutron radiation fields, however, none of them has network capabilities. In addition to preventing the smuggling of special nuclear material, this device can also alert an individual to the presence of harmful neutron and gamma radiation.

The objective of this project was to develop a networked gamma and neutron radiation detection system for use with special nuclear material tracking and surveillance technologies to provide real-time alarm detection and notification of unauthorized nuclear activities.

\section{DETECTION SYSTEM}

The detection system uses two sensors made of ${ }^{6} \mathrm{Li}$ and ${ }^{7} \mathrm{Li}$ loaded glass scintillators of $1 / 2$ " in diameter by 1 " long, which are mounted on small Hamamatsu photomultipliers. The large detector size makes it more sensitive to both neutrons and gamma rays. By exploiting the differences in the thermal neutron cross-section of ${ }^{6} \mathrm{Li}$ and ${ }^{7} \mathrm{Li}$, the device accurately measures both radiation fields separately as well as total radiation. Both isotopes are equally sensitive to gamma rays; however, the ${ }^{6} \mathrm{Li}$ detector is also sensitive to neutrons.

Fig. 1 shows the spectra taken with a ${ }^{252} \mathrm{Cf}$ source, which emits both gamma rays and neutrons simultaneously. As seen here, both ${ }^{7} \mathrm{Li}$ and ${ }^{6} \mathrm{Li}$ spectra are overlapping in the lowenergy region indicating that they are equally sensitive to gamma rays. The peak in ${ }^{6} \mathrm{Li}$ spectrum is due to the neutrons. By subtracting the ${ }^{7} \mathrm{Li}$ spectrum from the ${ }^{6} \mathrm{Li}$ spectrum one can obtain the pure neutron spectrum $\left({ }^{6} \mathrm{Li}-{ }^{7} \mathrm{Li}\right)$. Total counts are obtained by adding the counts in both spectra. Finally gamma ray counts are obtained by subtracting the neutron counts from the total counts. 


\section{SYSTEM COMPONENTS AND PROTOTYPE}

The system components shown in Fig. 2 consist of the ${ }^{6} \mathrm{Li}$ and ${ }^{7} \mathrm{Li}$ detectors mounted on two Hamamatsu R7400 photomultiplier tubes [6], the Cockroft-Walton voltage

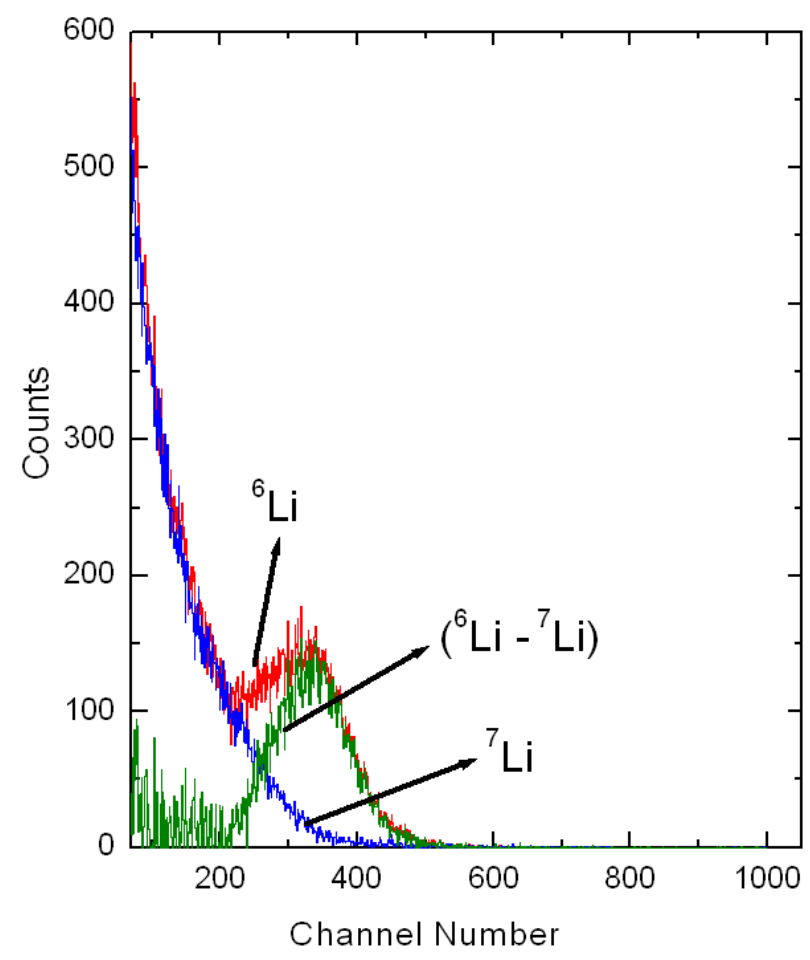

Fig. 1. ${ }^{6} \mathrm{Li},{ }^{7} \mathrm{Li}$, and $\left({ }^{6} \mathrm{Li}-{ }^{7} \mathrm{Li}\right)$ spectra.

multiplier board $(\mathrm{CW})$, the main circuit board, the LCD display, and firmware for the microcontroller.

The detector assemblies are connected to a high-voltage subsystem. The high-voltage subsystem output voltage is remotely or locally programmable with a range of up to about 800VDC through an Analog Devices AD5245 digital potentiometer. The $\mathrm{CW}$ voltage multiplier is made up of 9 stages. A voltage divider is inserted between the 8 th and 9 th stages to allow a $1 / 2$ voltage stage output (see ref. [7] for details). This voltage is applied to the anode of the PMTs. The $\mathrm{CW}$ high-voltage subsystem has been designed to operate with a supply voltage of 3.5VDC. The outputs of the PMTs are capacitively coupled with a pair of 1000VDC-rated capacitors and directed to an analog processing subsystem composed of a pair of amplifier circuits (an Analog Devices AD492) and a set of level discriminators (a TI TLC354C). The components of the analog subsystem have also been optimized to run with a supply voltage of $3.5 \mathrm{VDC}$. The amplifiers shape, filter, and amplify the low-level inputs generated by the PMTs. The gains for the amplifiers are controlled through a Dallas/Maxim DS1803-050 programmable potentiometer, which can be controlled remotely. The outputs of the amplifier circuits are directed to the set of level discriminators. A pair of discriminators is included for each amplifier output. Each of the discriminator levels is controlled through a Dallas/Maxim DS1803-100 remotely programmable digital potentiometer. The outputs of the discriminators are sent to the digital subsystem, which has also been optimized to operate with a supply voltage of 3.5VDC. The first stage of the digital subsystem is made up of a set of $74 \mathrm{HC} 123$ pulse generators. There is one pulse generator for each discriminator output for a total of four pulse generators. The outputs from the pulse generators are directed to a microcontroller. The microcontroller is a Microchip PIC18LF2525 and operates at an external frequency of $10 \mathrm{MHz}$ with a Linear Technology LTC6900 oscillator. The internal operational frequency of the microcontroller is set at $40 \mathrm{MHz}$ with a phase-locked loop (PLL) option. The microcontroller contains the code to count the pulses from the pulse generator, determine the rates and compare them to preset alarm thresholds, and then to notify the remote or local user of the alarm condition, if any have been exceeded. The microcontroller also interfaces to the communications subsystem, made up of an Intersil ICL3232 RS232 device, and the storage subsystem, made up of an STI M41T81 realtime clock chip and a Microchip 24LC256 EEPROM memory chip. The microcontroller is able to input commands and output data via the RS232 interface. The microcontroller also writes data directly to a Varitronix MDLS-16263-C-XLV-G-LED01G liquid crystal display. Power to the $\mathrm{CW}$ high-voltage subsystem is controlled from the microcontroller through a Linear Technology LTC1477 switch. A small piezo-crystal speaker is included for audible alarm annunciations.

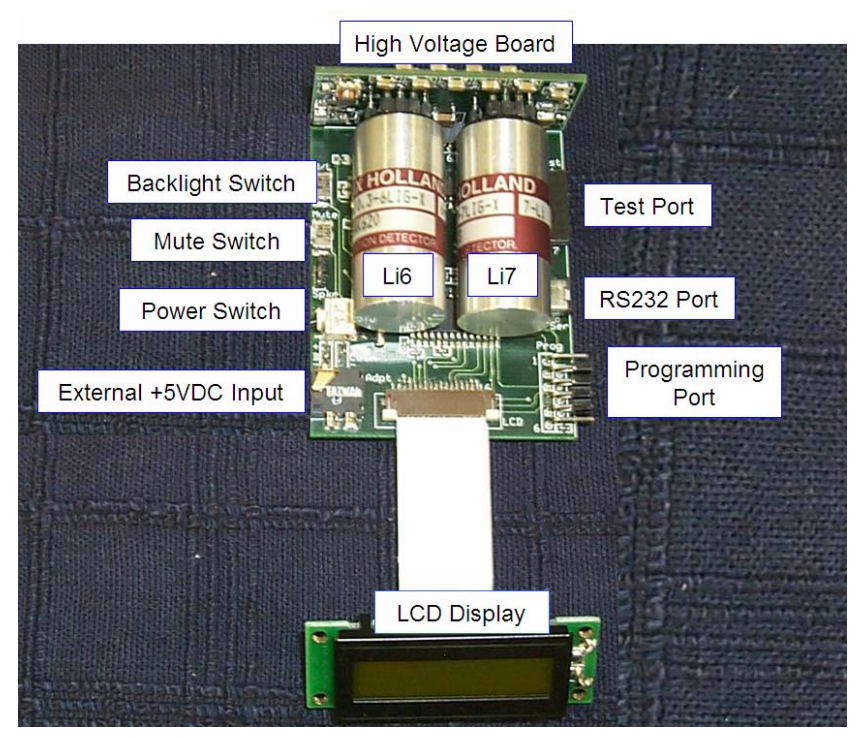

Fig. 2. Detection system Layout.

A serial interface was added to allow local or remote (through an optional radio-frequency (RF) link) command upload and data download capabilities. Configuration items such as gain settings and alarm threshold values can easily be changed now through a simple communications interface and the use of the programmable potentiometers. Other new components mounted on the board include a real time clock and a non-volatile memory. These components may be used to do accurate storage and time tagging of events. This data 
may be locally or remotely downloaded immediately or on a scheduled basis.

The previous detection system that we developed was about $4 "$ x 3" x 1.5" (see ref. [1] for details). The new prototype unit, shown in Fig. 3, is about 2.0" x 2.5" x 1.0" and was designed with an emphasis on compactness, recognizing the widespread need for a radiation detection instrument that provides both neutron and gamma ray detection in a single, portable unit. This radiation detection system can detect both gamma rays and neutrons in a radiation field as low as 10 $\mathrm{uR} / \mathrm{hr}$ above the ambient background. The system is designed to incorporate multiple devices in a distributed architecture with a central facility communicating through an RF link. The devices are independently capable of storing time-tagged radiation data locally in the event of an RF communications link failure. When the RF link is restored, the locally stored

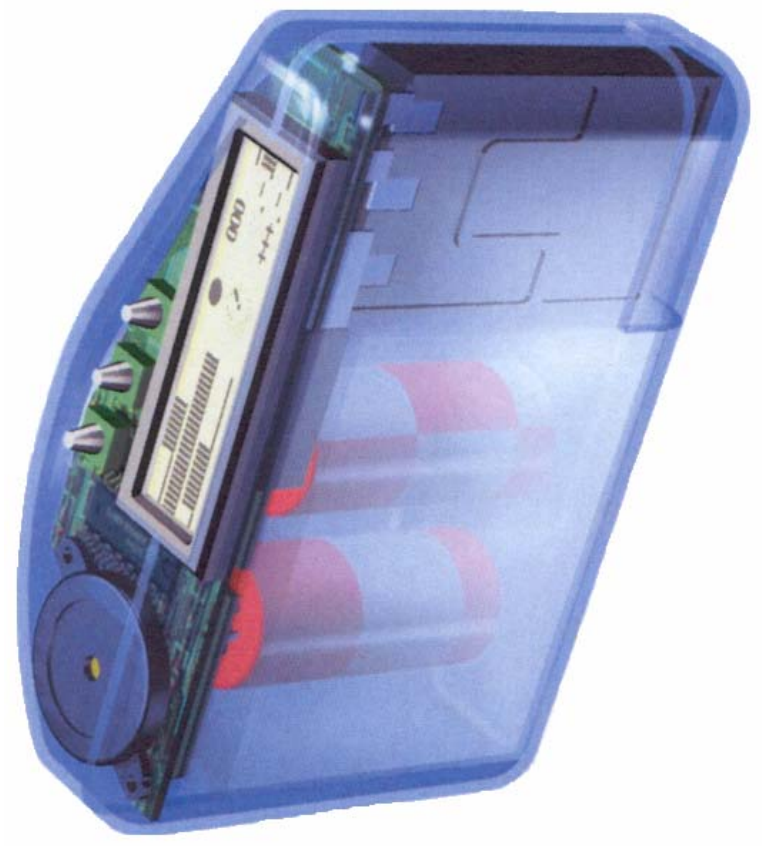

Fig. 3. Conceptual design of the prototype compact gamma and neutron detection system.

data can be downloaded to the central facility.

\section{NETWORK RF LINK CONSTRUCTION AND OPERATION}

An external interface board as shown in Fig. 4 was designed and built to translate the RS232 command interface from the NORMS device into a format suitable for a $900 \mathrm{MHz}$ RF wireless transceiver link to a remote PC. The interface board could either obtain power from the NORMS board (through the communications interface cable) or through an external 5VDC power supply. The selection was via a jumper on the interface board. The transceivers provided a "transparent" link from a remote PC to the NORMS device. The range of the transceivers was about 300 feet in an indoor environment with several obstructions to the RF path or about 1000 feet in an outdoor, unobstructed, line-of-sight environment. The power level of the transceivers and the frequency of operation were well within the limits of the ISM (Instrumentation, Scientific, and Medical) license free band, as specified by the

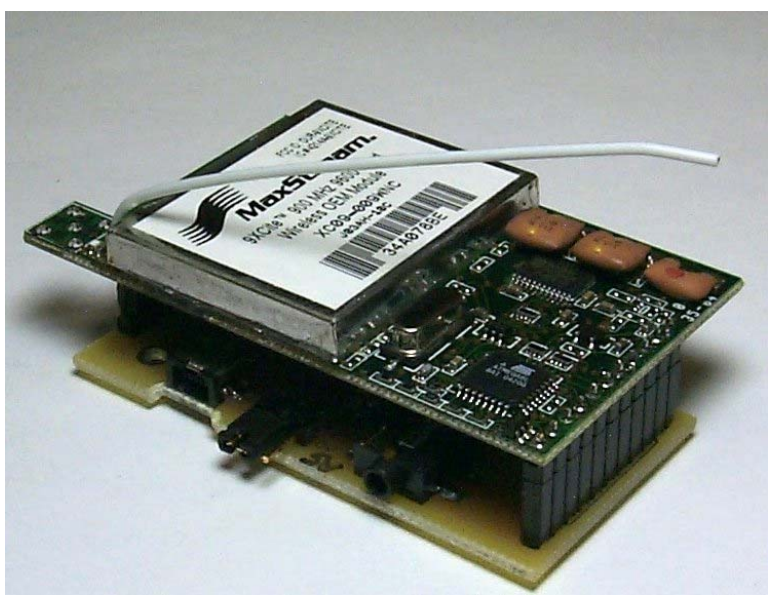

Fig. 4. RF and interface board.

FCC.

Basic tests were conducted using a "base station" attached to a local PC using one of the $900 \mathrm{MHz}$ transceivers. The NORMS device was connected through a cable to the external RF interface board. The external interface board contained another of the $900 \mathrm{MHz}$ transceivers. This constituted the "remote station". Preliminary communications were verified while the "base station" and "remote station" were about 6 feet away from each other. The "remote station" was then moved down a hallway within a building with many obstructions (metal walls, filing cabinets, etc) and

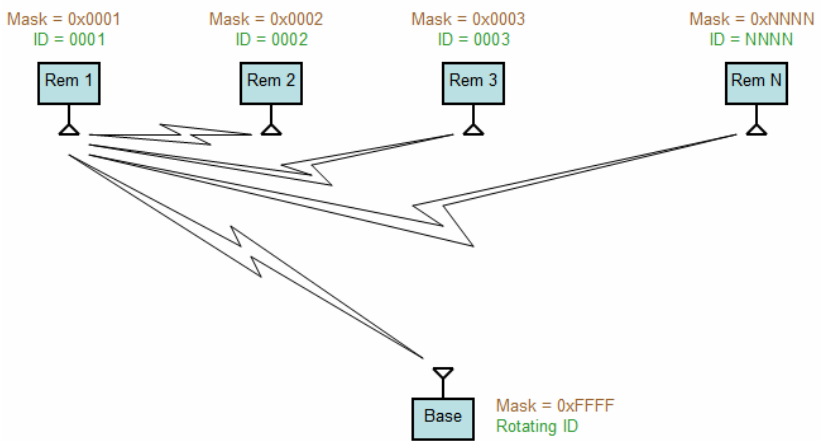

Fig. 5. NORMS RF Network Setup.

communications were continually tested and verified. The "remote station" was able to be moved to a location within the building approximately 125 feet away before communications began to start dropping out. At this point, the RF transmission had to penetrate at least four walls, doors, and several RF obstructions (metal filing cabinets, shielding in the walls, standing raw metal pieces, etc).

The RF communications system is designed to operate with one "base station" and several "remote stations". A typical example is shown in Fig. 5. The base station" controls all communications (normally) by polling each of the "remote stations", in turn and waiting for the reply. A simple ID code 
is included in the communications protocol to ensure only the selected "remote station" responds to the poll request. Likewise, the ID included in the reply from the "remote station" to the "base station" ensures only the "base station" accepts the response. In the case of the "remote station" losing communication with the "base station" (as determined by the "remote station" not sensing a poll request within a specific time and the "base station" not getting a response to the poll request within a specific time), the "remote station" goes into a stand-alone operation. The "remote station" will store all data obtained to local memory and await the reestablishment of the communications link. At this point the "remote station" can download the history of locally stored data to the "base station".

The device is designed to also allow a local connection to a device such as a PDA for portable applications. The device is small enough to easily fit in a shirt pocket and will run for 2 days on its internal battery. The device incorporates a method of allowing all system parameters to be controlled remotely. This allows a central facility to modify alarm set points on a device by device basis dynamically.

\section{SYSTEM FUNCTIONALITY AND APPLICATION}

The product focus is on modular functionality, which can be used in several operational modes, including as a standalone data collector, as an interactive device indicating a "normal/alarm" condition, or as a more detailed interactive device used for directional pinpointing of nuclear sources with emphasis on speed of detection. The device is extremely small but very sensitive. A logistic store of a few of these devices will allow easy interchangeability based on the specific application. The same device could be set to act as a member of a large distributed group to detect movements in a storage facility via RF link to central facility or could be set to interface to a PDA (either wired or wirelessly) giving a first responder an indication of a radiation hazard prior to entering a scene. Logistics spares are minimized because the same unit can be used for multiple purposes.

This unit will have multiple applications within the national and international safeguards as well as homeland security communities. This device has been already incorporated into INL explosives detection system (IEDS) for monitoring two neutron generators used in this system. In addition, this sensor can be also incorporated into the future INL compact WMD proliferation detection system. The device is small enough to be used by first responders. Another advantage of this device with the integrated display and audio alarm is that it can notify the first responder of a potential radiation hazard prior to being exposed. Two typical modes of operation are described below.

\section{a) Scenario 1 for stand-alone system:}

The device would be worn on a belt or carried in a shirt pocket with the display facing up, toward the user. The device would be conditioned to alert the user in the event preset level thresholds were exceeded by way of an audible alert or a visual alarm like flashing the LCD screen. In addition, the display would give an indication to the user of the continuously changing levels of both neutron and gamma ray radiation by way of a signal strength meter, easily interpreted as a set of changing bars. The device could then be used to further find the exact location of the source by the radiation field strength as indicated on the display. Another example in this scenario is for Customs application and port entries where cargo containers are constantly arriving and leaving. An audible alarm would be activated if preset radiation levels are exceeded. Proper response authorities could then be notified to further isolate the source. A relative measure of strength as indicated on the device could be relayed to those authorities to give an indication of the severity and response method to be taken.

\section{b) Scenario 2 for remote monitoring of multiple radiation detection devices that are placed at strategic choke points in nuclear storage facilities:}

Multiple devices could be installed at different innocuous locations throughout the storage facility, each connected by way of a short-range radio frequency transceiver to a remote monitoring station. Each device could be tailored to look for different field strength alarm thresholds, depending on the specific installation. Some typical choke points might include the entrance or exit from escalators and general and emergency entrance and exit doors. Devices could also be installed in the ceiling. As an installed device detects a potential threat, a radio frequency message will be relayed to the remote monitoring station. The monitoring station then indicates the threat through audible alarms and/or a flashing screen for the operator to take an appropriate action.

Some other typical applications include the tracking of sources within a facility, the interaction to a PDA/smart phone for data logging and worldwide control, and the detection of neutron sources hidden within medical isotopes.

\section{FUTURE ENHANCEMENTS}

The following is the list of modifications and enhancements that will be implemented.

- Increase sensitivity of gamma/neutron detectors.

- Modifications to include a memory chip to capture video and radiation data.

- Integration of commercially available video surveillance systems. This would be a WI-FI-based video single or multiple camera systems with remotely controlled tilt/pan/rotate/zoom functions. 


\section{CONCLUSION}

We have designed and built a pocket radiation detection system with network capabilities (NORMS), which can detect both neutrons and gamma rays instantly. It can detect small amounts of shielded plutonium, plutonium hidden inside medical isotopes, and low-level neutrons in a very strong gamma-ray field. This system operates on one rechargeable Li-ion battery and is small enough to be put in a pocket or worn as a clip-on for a stand-alone operation. However, its main application is to be used as a networked radiation detection system for providing an autonomous, traceable, and secure method of tracking radioactive materials within a secure environment, such as DOE materials handling facilities.

The network capability of the NORMS provides a platform to continuously monitor a critical area for abnormal conditions. The area may be a nuclear storage facility, a shopping mall, a large building, etc, each with multiple entry/exit points to be monitored. If any device in the network detects a condition whereby the alarm setpoints are exceeded, a remotely-connected base location will be immediately notified. The remote location will log normal and abnormal conditions. In the event of an alarm condition, a proper response will be initiated. The response could be calling a preset phone notification list, notifying a roaming security officer, turning an alarm monitor light on a console to red, etc. While the remotely located devices are designed to operate in the connected mode of operation, in the event communications is lost with the base location, no data will be lost. Data will be stored locally and can be downloaded when the communications is restored. The versatility of the system allows for dynamic reprogramming of device alarm limits, etc.

\section{REFERENCES}

[1] R. Aryaeinejad and D.F. Spencer, "Pocket Dual Neutron/Gamma Radiation Detector," IEEE Trans. Nucl. Sci., vol. 51, no. 4, pp. 16671671, August 2004.

[2] R. Aryaeinejad, Y.X. Dardenne, J.D. Cole, and A.J. Caffrey, "Palm-size Low-level Neutron Sensor for Radiation Monitoring," IEEE Trans. Nucl. Sci., vol. 43, no. 3, pp. 1539-1543, June 1996.

[3] G.L. Jensen, J.B. Czirr, Nucl. Instrum. Methods, vol. 205, pp. 461-463, 1983.

[4] Sensor Technology Engineering, Incorporated, 55533 Hollister Ave. \#1, Santa Barbara, CA 93117, USA.

[5] Thermo Electron corporation, Radiation Measurement \& Protection, 504 Airport Road, Santa Fe, NM 87507 ,USA. [Online]. Available: www.thermo.com.

[6] Hamamatsu Corporation, 360 Foothill Road, P.O. Box 6910, Bridgewater, NJ 08807-0910, USA. [Online]. Available: www.hamamatsu.com.

[7] D.F. Spencer, R. Aryaeinejad, and Edward Reber, "Using the CockroftWalton Voltage Multiplier with small photomultipliers," IEEE Trans. Nucl. Sci., Vol. 49, No. 3, pp. 1150-1153, June 2002. 